\title{
Impact on medical students of incorporating GALS screen teaching into the medical school curriculum
}

\author{
R A Fox, J E Dacre, C L Ingham Clark, A D Scotland
}

\begin{abstract}
Objective-To assess the impact of GALS locomotor screen teaching to all 3 rd year medical students, at a British medical school.

Method-In 1998, during their 3rd year, all students were taught the GALS screen in a one hour small group session. At the end of this year, 242 medical students undertook a 16 station Objective Structured Clinical Examination (OSCE). One station assessed the locomotor screening examination, while six stations assessed the examination of other systems. The students completed a five point likert scale, self rating their confidence in each of the skills assessed at this time. Preregistration house officers (PRHOs) at two London hospitals were invited to undertake the same $O S C E$ and self rating. Results-The students performed the locomotor screen well (mean station score $80 \%$ ). Three body systems were examined better and one significantly worse $(p<0.05)$. 22/40 PRHOs undertook the assessment. Compared with the students they examined the locomotor system (mean score $20 \%, \mathrm{p}<0.001$ ), but not other systems, less well. The PRHOs felt less confident $(p<0.05)$ examining the locomotor system (mean rating 3.6/5) than the other systems (mean rating 4.6/5), while no significant difference in confidence ratings was seen for the students.

Conclusion-Students who are taught the GALS screen as part of the curriculum, perform it well in an end of year OSCE, as confidently as other systems, and to a higher standard than PRHOs. Further study is required to determine whether this benefit persists, overcoming the poor skills and confidence in locomotor examination of existing PRHOs, not previously taught a GALS screen.

(Ann Rheum Dis 2000;59:668-671)
\end{abstract}

Musculoskeletal disorders are commonly seen in general practice ${ }^{1}$ and in hospital inpatients. ${ }^{23}$ Junior doctors appear to be poor at detecting such disorders, and it has been suggested that this is owing to a combination of poor skills and their failure to assess the locomotor system in general medical patients. ${ }^{2-5}$

Traditionally, medical students have seen the locomotor system as complex and difficult to examine. The GALS locomotor system screen ${ }^{5}$ is a quick, easy to perform, sensitive indicator of locomotor abnormality ${ }^{6}$ and has been shown to be a reliable and valid measure of functional ability. ${ }^{7}$ It can be quickly learnt by undergraduates ${ }^{5}$ and postgraduates. ${ }^{8}$ The General Medical Council of Great Britain gives a high priority to the acquisition of basic clinical skills $^{9}$ and it has been suggested that the GALS screen should be included in the undergraduate curriculum ${ }^{5}$ in the UK.

The impact of teaching the GALS screen routinely as part of the curriculum has not formally been evaluated. We have examined the effect of GALS screen teaching to all 3rd year University College London medical students by incorporating it into an end of year summative assessment, which is mandatory for all these students. There are three main questions addressed: Are these students learning the GALS screen? How do they perform this screen compared with their ability to examine other body systems? How able are existing preregistration house officers to perform a locomotor examination?

\section{Method}

MEDICAL STUDENTS

All 3rd year medical students at University College London medical school, UK, are now taught the GALS screen. This consists of two screening questions: "Do you have any pain or stiffness anywhere?" and "Do you have any difficulty washing, dressing, or climbing stairs/ steps?" as well as the 11 examination tasks shown in table 2 . This teaching session takes place during their general practice or rheumatology firm attachment, which may occur anytime between October and July of an academic year depending on when the student is allocated to these two firms (students rotate every six weeks). The GALS teaching typically is incorporated into part of a one hour small group session. Those 242 medical students completing their third year in July 1998, undertook a 16 station Objective Structured Clinical Examination (OSCE) of basic clinical skills at this time.

The students were asked to complete a named questionnaire rating their confidence in examining each of the body systems assessed in the OSCE on a five point likert scale (ranging from not at all (1) to very (5)). The rating questionnaire was completed immediately before or immediately after the OSCE (before any exam feedback).

PRE-REGISTRATION HOUSE OFFICERS (PRHOS)

The 40 PRHOs working at the Chelsea and Westminster and Whittington hospitals during
Accepted for publication 29 June 2000 
July 1998 were invited to undertake the same OSCE as the medical students. Participation was voluntary and was part of a larger study looking at PRHO clinical skills. Immediately before the OSCE the PRHOs completed the same questionnaire as the students, asking them to rate their confidence in the physical examination skills to be assessed. The PRHOs had all been taught rheumatology as undergraduates but had not been taught the GALS screen specifically.

OSCE

An OSCE is a carousel, made up of clinical tasks or "stations", and has been shown to be a good test of clinical competence. ${ }^{10}$ In this OSCE each station typically lasted for five minutes, during which the student was given written instructions for a standardised task. The student then performed the task (for example, taking a short history from a patient or examining the patient) while an observer rated his performance against a standardised marking schedule. After completing a station the student went to the next one assessing a different task. The observers were mostly clinicians or, in some cases, nurses. Rheumatology consultants or specialist registrars marked the GALS screen station. In order for all the students to undertake the OSCE, the same 90 minute circuit was run concurrently four times a day for two days at two different sites. The observers were trained for 45 minutes on the part of the OSCE circuit in which they were involved. Seven of the 16 OSCE stations assessed the examination of body systems, including one asking the candidates to "perform a screening locomotor system examination (for example, GALS) on a patient complaining of joint pain and stiffness". Each station consisted of 9-13 items, each of which is marked pass ( $1 \mathrm{mark}) /$ fail or not done (0 marks). A total mark for each station was obtained by standardising item scores to give a maximum mark of 20 for each station. Each OSCE station was also marked with a global score (fail/borderline fail/borderline pass/pass), for which the examiner used his judgment of overall performance irrespective of the item score.

STATISTICS

The data were analysed using SPSS for MS windows. The data rendered non-parametric testing most appropriate to compare groups for significant differences. The Wilcoxon paired rank sum test was used to compare the performance of medical students on different OSCE stations and their confidence ratings. A Mann-Whitney $U$ test was used to compare PRHO and medical student performance. A Bonferroni correction was used where multiple comparisons were performed.

\section{Results}

22/40 PRHOs and 242 medical students undertook the OSCE. All PRHOs and 196 (81\%) medical students completed the confidence rating questionnaire.
Table 1 Performance of 242 medical students on the seven physical examination OSCE stations

\begin{tabular}{ll}
\hline OSCE station & $\begin{array}{l}\text { Mean station score* } \\
\text { (95\% CI) }\end{array}$ \\
\hline Chest examination & $18.5(18.2$ to 18.8$)$ \\
Blood pressure & $17.4(16.9$ to 17.8$)$ \\
Abdominal examination & $17.3(17.0$ to 17.7$)$ \\
GALS screen & $16.0(15.5$ to 16.4$)$ \\
Neurology (sensation of legs) & $15.4(14.9$ to 15.8$)$ \\
Peripheral pulse examination & $14.1(13.6$ to 14.7$)$ \\
Higher mental function & $15.9(15.5$ to 16.3$)$ \\
\hline
\end{tabular}

^Maximum station score is 20 .

$\mathrm{CI}=$ confidence interval

ARE THE STUDENTS LEARNING TO PERFORM THE GALS SCREEN?

The medical students performed the screening locomotor system examination well, achieving a mean score of $16(80 \%)$, which is similar to the overall mean mark for all 16 stations $(78 \%)$.

COMPARISON OF MEDICAL STUDENT GALS SCREEN PERFORMANCE WITH THAT OF OTHER SYSTEMS EXAMINATION

Table 1 compares the performance of the GALS screening examination with that of the six other physical examination stations. Friedman's two way analysis of variance test suggests there are differences in the mean marks of the physical examination stations $(p<0.001)$. The GALS screen station results were significantly better than peripheral pulse examination, though not quite as good as respiratory/ abdominal examination or blood pressure measurement $(\mathrm{p}<0.05$ with Bonferroni correction). There were no significant differences between the performance of the GALS screen and neurological or higher mental function examination.

The medical students felt as confident examining the locomotor system (mean self confidence rating 3.9 (SD 0.9)) as other body systems (mean self confidence rating 3.9 (SD 0.6)). There was no significant difference in overall OSCE performance of medical students who did/did not complete the confidence questionnaire.

\section{PRHO SCREENING LOCOMOTOR SYSTEM}

EXAMINATION SKILLS

Unlike the medical students, the PRHOs felt significantly less confident (Wilcoxon paired rank sum test, $\mathrm{p}<0.05)$ examining the locomotor system (mean self confidence rating 3.6 (SD 1.1)) than other body systems (mean self confidence rating 4.6 (SD 0.5)). The PRHOs performed better than the students overall in the six other physical examination stations, though this did not reach significance (mean score $86 \%$ v 82\% respectively, p>0.05) but significantly worse in the locomotor examination screen $(5(20 \%) v 16(80 \%), \mathrm{p}<0.001)$. Table 2 shows the difference in PRHO and student performance for each of the 11 items assessed at the locomotor screening examination station. $88 \%$ of students and $0 \%$ of PRHOs achieved a pass/borderline pass for this station. Examiner feedback suggested that the PRHOs had little idea of how to examine the locomotor system. 
Table 2 Comparison of pre-registration house officers (PRHOs) and medical students in performing a screening locomotor examination

\begin{tabular}{lcc}
\hline & \multirow{2}{*}{ No of candidates achieving a pass (\%) } \\
\cline { 2 - 3 } OSCE marking schedule & PRHOs & Medical students \\
\hline Inspects the patient from the front, back, and side & $1(5)^{\star}$ & $150(62)$ \\
Asks the patient to walk and inspects their gait & $8(36)^{\star}$ & $237(98)$ \\
Asks the patient to show their hands, inspects and palpates MCP† joints & $11(50)^{\star}$ & $204(84)$ \\
Asks patients to make a power and precision grip & $8(36)^{\star}$ & $212(88)$ \\
Assesses radioulnar joints and elbow extension & $9(41)$ & $173(71)$ \\
Asks patient to put their hands behind their neck with elbows back to assess shoulders & $14(64)$ & $193(80)$ \\
Checks hip internal rotation while patient lies on the couch & $2(9)^{\star}$ & $175(72)$ \\
Looks for knee swelling, checks knee flexion, and feels for crepitus & $1(5)^{\star}$ & $169(70)$ \\
Has a quick look at the ankles and checks for MTPt joint tenderness/swelling & $0(0)^{\star}$ & $174(72)$ \\
Asks patient to bend forward and checks for lumbar flexion & $2(9)^{\star}$ & $223(92)$ \\
Checks for neck lateral flexion & $2(9)^{\star}$ & $207(86)$ \\
\hline
\end{tabular}

^PRHOs performed significantly worse than students $\left(\chi^{2} \mathrm{p}<0.05\right.$, with Bonferroni correction).

$\dagger \mathrm{MCP}=$ metacarpophalangeal; MTP = metatarsophalangeal.

OSCE

The overall reliability of the 16 station OSCE was 0.65 (Cronbach $\alpha$ ). There was no significant difference in the overall OSCE mark or the overall mark for the six physical examination stations (excluding the GALS screen) according to the site or the date of the OSCE. Students undertaking the OSCE on day 2 of testing had a slightly higher mean GALS screen station mark than those on day 1 (16.0 v 15.0, p<0.001), but there was no significant difference in scores between the two examination sites.

\section{Discussion}

GALS screen teaching during the 3rd year results in medical students performing a locomotor screening examination well in an end of year OSCE with a mean score $16(80 \%)$. This is similar to their ability to perform an examination of other body systems. The medical students felt as confident examining the locomotor system as the other body systems.

The PRHOs performed a screening locomotor examination badly, and none of them achieved a pass/borderline pass on this station. A global score was included to ensure that the PRHOs were not examining the joints well without adhering to a GALS-like protocol- in reality, from both the item and global scores, as well as the examiners' comments, the PRHOs had little idea how to examine the locomotor system. The PRHOs performed better (though this did not reach statistical significance) in the other physical examination stations, suggesting that this is a specific deficit in locomotor system examination ability and not just the PRHOs undertaking an inappropriate assessment designed to test basic clinical skills of medical students. Consistent with this, the PRHOs felt significantly less confident examining the locomotor system than the other systems. Although the generalisability of these findings is potentially limited by the small sample of PRHOs, we believe the PRHOs in this study to be at worst representative and probably better than the majority of their peers. This is because inclusion in the study was voluntary, the PRHOs had the confidence to allow their clinical skills to be assessed, and all these PRHOs were working in highly sought after posts in teaching hospitals. We do not know whether these PRHOs felt as unconfident examining the locomotor system as undergraduates or whether they had become deskilled from not performing locomotor examinations on patients seen in hospitals.

Traditional medical clinical examinations (long and short cases and orals) have been hampered by their unreliability. The OSCE has good psychometric properties and has better reliability than, for example, oral examinations for a given testing time duration. ${ }^{11}$ In particular, the student is given a standardised task to do on each station and the marking schedule is similarly standardised, with a view to reducing inter- and intra-observer error. Because the number of clinical tasks undertaken in an OSCE is much larger than traditional British clinical examinations, we could compare the performance of candidates in seven different physical examination stations.

This study was designed to evaluate the effect of incorporating GALS screen teaching into the curriculum. It was not a randomised study of the effect of teaching the GALS screen to a cohort of students in a controlled trial environment. Although such studies have their merits they tend to result in improved student performance because the students are aware that they are being studied" ("Hawthorn effect") and they do not evaluate the effect of teaching on learning in the uncontrolled real world-that is, the student educational environment. The drawback to standardisation in this examination was that the students performed the GALS screen better if they undertook the OSCE on day 2. This advantage for the day 2 candidates might have been due to them revising the GALS screen overnight. The rigid checklist nature of the GALS screen does make this easy to do. Despite this, the day 1 candidates still performed the examination well with a mean score of $15(75 \%)$, suggesting the GALS screen has been learnt well by these 3rd year medical students. The fact that the GALS screen scores, but not most of the other station scores, improved on the second day of testing reinforces the ease with which it can be learnt, which is one of its desirable properties.

Using PRHOs as a control group is not ideal to assess the impact of GALS screen teaching to undergraduates. What it does tell us is that medical students recently taught the GALS screen examine the joints better than these PRHOs and that, currently, PRHOs examine 
the locomotor system poorly. Ideally, to assess the impact of GALS screen teaching directly, half of the medical students should have been taught it and the other half taught by traditional rheumatology teaching methods. Currently, in view of the perceived benefit of the GALS screen, it is taught to all our medical students. An alternative would be to compare student performance in an OSCE from two medical schools, only one of which teaches the GALS screen. Such a study would be possible, but it is always difficult to control for other curricular differences between the medical schools.

The students in this study will have been taught the GALS screen up to nine months before the OSCE, depending on when they undertook the GP/rheumatology firms. Although we have shown that medical students can be taught to perform the GALS screen well, the duration of this benefit is uncertain. One small study has shown that one 15 minute GALS screen teaching session increases the likelihood of the locomotor system being examined by junior doctors on hospital inpatients six weeks later. ${ }^{8}$ Further study is required to see if these medical students retain their confidence and ability to perform a locomotor screening examination when they become PRHOs and beyond, and to see if this confidence is associated with improved diagnostic acumen of rheumatological conditions.
We thank the pre-registration house officers who agreed to participate in this study.

1 Wright V. The epidemiology of disability. J R Coll Physicians Lond 1982;16:178-83.

2 Doherty M, Abawi J, Pattrick M. Audit of medical inpatient examination: a cry from the joint. J R Coll Physicians Lond 1990;24:115-18.

3 Fox R, Luder R, Sinclair H. Rheumatological assessment in hospitalised elderly medical patients [abstract]. Age and Aging 1996; suppl 2: abstr 25.

4 Spencer MA, Dixon AS. Rheumatological features of patients admitted as emergencies to acute general medical wards. Rheumatol Rehabil 1981;20:71-3.

5 Doherty M, Dacre J, Dieppe P, Snaith M. The (GALS) locomotor screen. Ann Rheum Dis 1992;51:1165-9.

6 Jones A, Ledingham J, Regan M, Doherty M. A proposed minimal rheumatological screening history and examination: the joint answers back. J R Coll Physicians Lond 1991;25:111-15.

7 Plant MJ, Linton S, Dodd E, Jones PW, Dawes PT. The GALS locomotor screen and disability. Ann Rheum Dis 1993;52:886-90.

8 Jones A, Regan M, Ledingham J, Doherty, M. Can we alter doctors' awareness of locomotor problems [abstract]. Br J Rheumatol 1991; suppl: abstr 30.

9 General Medical Council Education Committee. Tomorrow's doctors. Recommendations on undergraduate medical education. London: General Medical Council, 1993.

10 Cohen R, Rothman AI. A comprehensive assessment of graduates of foreign medical schools. Annals of the Royal College of Physicians and Surgeons of Canada 1988;21: 505-9.

11 Van der Vleuten C, Newble D, Case S, Holsgrove G, McCann B, McRae C, et al. Method of assessment in certification. In: Newble D, Jolly B, Wakeford R, eds. The certification and recertification of doctors: issues in the assessment of clinical competence. Cambridge: Cambridge University Press, 1994:105-25.

12 Roethlisberger FJ, Dickson WJ. Management and the worker. Cambridge, MA: Harvard University Press, 1939. 